\title{
Ranking Function to Solve a Fuzzy Multiple Objective Function
}

\section{Iden Hasan Hussein}

\author{
Rasha Jalal Mitlif ${ }^{2 *}$
}

${ }^{1}$ Department of Mathematics, College of Science of Woman, University of Baghdad, Baghdad, Iraq.

${ }^{2}$ Department of Applied Sciences, Branch of Mathematics and Computer Applications, University of Technology, Baghdad, Iraq.

*Corresponding author: idenalkanani58@gmail.com, 10096@uotechnology.edu.iq *

*ORCID ID: https://Orcid.org/0000-00015033-8753, https://orcid.org/0000-0002-6492-8196*

Received 8/8/2019, Accepted 16/2/2020, Published Online First 6/12/2020, Published 1/3/2021

This work is licensed under a Creative Commons Attribution 4.0 International License.

\begin{abstract}
:
In this paper two ranking functions are employed to treat the fuzzy multiple objective (FMO) programming model, then using two kinds of membership function, the first one is trapezoidal fuzzy (TF) ordinary membership function, the second one is trapezoidal fuzzy weighted membership function. When the objective function is fuzzy, then should transform and shrinkage the fuzzy model to traditional model, finally solving these models to know which one is better.
\end{abstract}

Key words: Fuzzy multiple objective (FMO), Ranking function (RF), Trapezoidal fuzzy (TF) number.

\section{Introduction:}

The most important techniques in operational research are fuzzy programming problems, in which many real data in the programming model deal with vague numbers and variables by using fuzzy programming models. Bellman and Zadeh (1) suggested the fuzzy decision making. The fuzzy programming problem is focused by Zimmermann (2) making application in fuzzy multiple objective programming techniques Maleki (3) suggested many methods to find the solution for the programming model. Iden and Anfal (4) used an algorithm of ranking function (RF) to solve fuzzy transportation problem. Nehi and Hajmohamadi (5) employed ranking function (RF) method to solve the fuzzy multi objective (FMO) programming problem. Porcheivi and Vasanthi (6) used a new method to treat the fuzzy variable programming problems (PP) based on ranking function (RF) for multiple objective function. Kiruthiga and Loganathan (7) employed the fuzzy multi objective programming problems (FMOPP) by fuzzy programming model and reduced it to crisp model. Mitlif (8) used an efficient method to solve the fully fuzzy multi objective programming problems (FFMOPP). Iden and Abood (9) used fully fuzzy games problems while there is uncertainty in data by applying the fuzzy payoffs problem by using three different ranking functions. Ingle and Ghadle (10) used a new ranking function method to solve the fully fuzzy programming problems (FFPP) with hexagonal (HFN).

The aim of this paper is employing two ranking functions (RF) for fuzzy variable in multiple objective functions by using trapezoidal fuzzy (TF) membership function when the objective variables are fuzzy. This paper presents in section two multiple objective programming (MOP). In section three the concepts of fuzzy theory and definitions are introduced. Section four deals with the triangular fuzzy number. In section five the fuzzy multiple objective problem is tackled. Section six presents ranking function of triangular fuzzy number and algorithms of ranking function (RF), and section seven involves the test example. Finally, section eight presents conclusions.

\section{Multiple Objective Programming (MOP)}

This section describes many decision problems, which have multiple objectives to be optimized simultaneously. If the constraints of the problems and its multiple objectives can be expressed as functions, then it is called as a MOP problem. The basic approach to solve multi objective problems is to determine a solution that represents an acceptable trade - off, compromise between the objectives, or determine a set of such solutions that allow the decision marker to choose among them. 
Defining the multi objective function problem as follows: there are $\mathrm{P}$ cost vectors denoted by $c_{k}=$ $\left(c_{k 1}, \ldots, c_{k n}\right), k=1, \ldots, p$, the usual set $m \times n$ constraints $A T=b$ and $T \geq 0$. Each $c_{k} T_{i}$ where $i=1, \ldots, n$ defines the objective function, subject to the constraints; the problem is stated as below:

"Maximize" $C_{\tau}$

Subject to the constraint

$A T=b$

$T \geq 0$

where $\quad C=\left(\begin{array}{c}c_{1} \\ \vdots \\ \cdot \\ c_{p}\end{array}\right)=\left(\begin{array}{ccc}c_{11} & \ldots \ldots . & c_{1 n} \\ c_{21} & \ldots \ldots . & c_{2 n} \\ \cdot & \cdot & \cdot \\ \vdots & \vdots & \vdots \\ c_{p 1} & \ldots \ldots . & c_{p n}\end{array}\right)$

Definition 1 (11): $\tau^{o}$ is said to be an efficient solution to (1) if $\tau^{o}$ is feasible and no other feasible solution $T$ exists such that $C T \geq C T^{O}$ and $C T \neq$ $C T^{O}$.

Theorem 2 (11): let $C_{K} T$ be one of the objective function of the vector maximum (1). Suppose the programming problem of maximizing $C_{K} T$ together with $A T=b$ and $T \geq 0$ and let $T^{O}$ be its unique solution. Then $T$ is an efficient of (1).

\section{Concept of Fuzzy Theory}

Some definitions of fuzzy theory are listed throughout this section.

Definition 3 (12): Assume $T$ is a non-empty set, a fuzzy set $\tilde{A}$ in $T$ is characterized by the membership function $M_{\tilde{A}}=T \rightarrow[0,1]$ and $A(T)$ is explained as the degree of membership of element $T$ in $\tilde{A}, \tau$ $\epsilon T$.

Definition 4 (13): The crisp set of elements which belong to $\tilde{A}$ at least to the degree $\alpha$ is named as $\alpha$ level set

$A_{\alpha}=\left\{\tau \in T / M_{\tilde{A}} \geq \alpha\right\}$.

$\hat{A_{\alpha}}=\left\{\tau \in T / M_{\tilde{A}}>\alpha\right\}$ is called strong $\alpha-$ level set or strong $\alpha$ - cut.

Definition 5 (14): $\tilde{A}$ is a fuzzy set on $R$ which satisfy the conditions below:

1- There exists at least one $\tau_{0} \in R$ with $M_{\tilde{A}}\left(T_{0}\right)=$ 1.

2- $\mathrm{M}_{\widetilde{\mathrm{A}}}(\mathrm{T})$ is piecewise continuous.

3- A is convex and normal.

Triangular Fuzzy Number (TFN)

A fuzzy number (FN) $\tilde{A}=(a, b, c)$ in $\mathrm{R}$ is said to be a TFN if its membership function $M_{A}: \mathrm{R} \rightarrow[0,1]$ possesses the characteristic below:

$$
M_{A} T=\left\{\begin{array}{lc}
\frac{\tau-a}{b-a} & a \leq \tau \leq b \\
1 & \tau=b \\
\frac{c-\tau}{c-b} & b \leq \tau \leq c
\end{array}\right.
$$

where $\mathrm{a}, \mathrm{b}, \mathrm{c}$ are core $(A)$, left width and right width respectively.

\section{Fuzzy Multiple Objective Problem (FMOP)}

The fuzzy multiple objective problems are the multiple objective problems with triangular fuzzy quantities. Now the formula of the fuzzy objective function problem is described by:

Maximize z (Minimize $z)=\left(\begin{array}{c}\tilde{C}_{1} T \\ \tilde{C}_{2} T \\ \vdots \\ \tilde{C_{P} T}\end{array}\right)$

Subject to

$A T=b$

$T \geq 0$

\section{Ranking Function of Triangular Fuzzy Number} (15):

The function $R: F(M) \rightarrow R$ is RF which maps each FN to $R$; where $F(M)$ represents the set of TFN.

Assume that A and B are TFN. The following identities are satisfied

$1-A<B$ iff $R(A)<R(B)$.

$2-B<A$ iff $R(B)<R(A)$.

$3-A \approx B$ iff $R(A) \approx R(B)$.

$4-A-B=0$ iff $R(A)-R(B)=0$.

The TFN $\tilde{A}=(a, b, c)$ in $F(M)$ where $R(\tilde{A}) \geq 0$ is said to be positive and denoted by $\tilde{A}>0$.

\section{Ranking Function Algorithms: \\ - Algorithm 1}

We use the previous triangular membership function and by employing the $\alpha$ - cut where $\alpha \in[0,1]$ then

$\frac{\tau-a}{b-a}=\alpha \rightarrow \tau=a+(b-a) \alpha=\inf \tilde{A}(\alpha)$.

$\frac{c-\tau}{c-b}=\alpha \rightarrow \tau=c-(c-b) \alpha=\sup \tilde{A}(\alpha)$.

Applying the ranking function.

$R(\tilde{A})=\frac{1}{2} \int_{0}^{1}[\inf \tilde{A}(\alpha)+\sup \tilde{A}(\alpha)] d \alpha$

$\alpha \in[0,1]$ then $R(\tilde{A})$ becomes the following equation:

$$
\begin{aligned}
R(\tilde{A})= & =\frac{1}{2} \int_{0}^{1}[a+(b-a) \alpha+c-(c \\
-b) \alpha] d \alpha & \\
R(\tilde{A})= & =\frac{1}{2} \int_{0}^{1}[a+2 \alpha b-\alpha a+c-\alpha c] d \alpha \\
R(\tilde{A})= & \frac{1}{4}[a+2 b+c]
\end{aligned}
$$

\section{- Algorithm 2}

In this algorithm, we used the weighted triangular membership function, will be used below: 
$M_{\tilde{A}}(T)=\left\{\begin{array}{lc}\frac{w(\tau-a)}{b-a} & a \leq \tau \leq b \\ w & \tau=b \\ \frac{w(c-\tau)}{c-b} & b \leq \tau \leq c\end{array}\right.$

By using the $\alpha-$ cut where $0 \leq w \leq 1$ then

$$
\begin{aligned}
\frac{w(\tau-a)}{b-a}=\alpha & \rightarrow \tau=a+\frac{\alpha}{w}(b-a) \\
& =\inf \tilde{A}(\alpha) . \\
\frac{w(c-\tau)}{c-b}=\alpha & \rightarrow \tau=c-\frac{\alpha}{w}(c-b) \\
& =\sup \tilde{A}(\alpha) .
\end{aligned}
$$

Applying the RF, $\alpha \in[0, w]$

$$
\begin{array}{r}
R(\tilde{A})=\frac{1}{2} \int_{0}^{w}[\inf \tilde{A}(\alpha)+\sup \tilde{A}(\alpha)] d \alpha \\
R(\tilde{A})=\frac{1}{2} \int_{0}^{w}\left[a+\frac{\alpha}{w}(b-a)+c-\frac{\alpha}{w}(c\right. \\
-b)] d \alpha \\
R(\tilde{A})=\frac{1}{2}\left[\left.a \alpha\right|_{0} ^{w}+\left.\frac{\alpha^{2}}{2 w}\right|_{0} ^{w}(b-a)+\left.c \alpha\right|_{0} ^{w}\right. \\
\left.\quad-\left.\frac{\alpha^{2}}{2 w}\right|_{0} ^{w}(c-b)\right]
\end{array}
$$

\section{Numerical Examples}

Now two examples for fuzzy MOP problems are considered in this section:

\section{Example 1:}

$$
\begin{aligned}
& \text { Max } z_{1}=3 \tau_{1}+\tau_{2}+2 \tau_{3}+\tau_{4} \\
& \text { Max } z_{2}=\tau_{1}-\tau_{2}+2 \tau_{3}+4 \tau_{4} \\
& \text { Max } z_{3}=-\tau_{1}+5 \tau_{2}+\tau_{3}+2 \tau_{4} \\
& \text { Subject to } \\
& 2 \tau_{1}+\tau_{2}+4 \tau_{3}+3 \tau_{4} \leq 60 \\
& 3 \tau_{1}+4 \tau_{2}+\tau_{3}+2 \tau_{4} \leq 60 \\
& \tau_{1}, \tau_{2}, \tau_{3}, \tau_{4} \geq 0 \\
& \text { First: When we solve the crisp (MOP) problem one } \\
& \text { can obtain the following optimal solution } \\
& \text { Max } z_{1}=66, \quad \tau_{1}=18, \quad \tau_{2}=0, \\
& \text { Max } z_{2}=80, \quad \tau_{3}=6, \quad \tau_{4}=0 . \\
& \text { Max } z_{3}=75, \quad \tau_{3}=0, \quad \tau_{4}=20 . \\
& =0, \quad \tau_{1}=0, \quad \tau_{2}=0 .
\end{aligned}
$$

Second: Transforming the crisp multiple objective to fuzzy multiple objective and then solve them by two different ranking functions.

Now, we consider the multiple objective as triangular fuzzy numbers in the problem as follows: Let $\Delta_{1}=0.5$ and $\Delta_{2}=1$ where $\left(c_{i j}-\Delta_{1}, c_{i j}, c_{i j}\right.$ $\left.+\Delta_{2}\right), i=1,2,3, j=1,2,3,4$.

Max $z_{1}=(2.5,3,4) \tau_{1}+(0.5,1,2) \tau_{2}$

$$
+(1.5,2,3) \tau_{3}+(0.5,1,2) \tau_{4}
$$

$\operatorname{Max} z_{2}=(0.5,1,2) \tau_{1}-(0.5,1,2) \tau_{2}$

$$
+(1.5,2,3) \tau_{3}+(3.5,4,5) \tau_{4}
$$

$\operatorname{Max} z_{3}=-(0.5,1,2) \tau_{1}+(4.5,5,6) \tau_{2}$

Subject to

$$
+(0.5,1,2) \tau_{3}+(1.5,2,3) \tau_{4}
$$

$$
\begin{aligned}
& 2 \tau_{1}+\tau_{2}+4 \tau_{3}+3 \tau_{4} \leq 60 \\
& 3 \tau_{1}+4 \tau_{2}+\tau_{3}+2 \tau_{4} \leq 60 \\
& \tau_{1}, \tau_{2}, \tau_{3}, \tau_{4} \geq 0
\end{aligned}
$$

1. By applying algorithm 1 function $R(\tilde{A})=$ $\frac{1}{4}[a+2 b+c]$ then the multiple objective as follows:

$$
\begin{aligned}
& \operatorname{Max} z_{1}=3.125 \tau_{1}+1.125 \tau_{2}+2.125 \tau_{3} \\
& +1.125 \tau_{4} \\
& \operatorname{Max} z_{2}=1.125 \tau_{1}-1.125 \tau_{2}+2.125 \tau_{3} \\
& +4.125 \tau_{4} \\
& \text { Max } z_{3}=-1.125 \tau_{1}+5.125 \tau_{2}+1.125 \tau_{3} \\
& +2.125 \tau_{4}
\end{aligned}
$$

Subject to

$$
\begin{aligned}
& 2 \tau_{1}+\tau_{2}+4 \tau_{3}+3 \tau_{4} \leq 60 \\
& 3 \tau_{1}+4 \tau_{2}+\tau_{3}+2 \tau_{4} \leq 60 \\
& \tau_{1}, \tau_{2}, \tau_{3}, \tau_{4} \geq 0
\end{aligned}
$$

Solving the crisp multi objective to get the optimal solution

$$
\begin{gathered}
\text { Max } z_{1}=69, \quad \tau_{1}=18, \quad \tau_{2}=0, \\
\tau_{3}=6, \quad \tau_{4}=0 . \\
\operatorname{Max} z_{2}=82.5, \tau_{1}=0, \quad \tau_{2}=0, \\
\tau_{3}=0, \quad \tau_{4}=20 . \\
\text { Max } z_{3}=76.87, \quad \tau_{1}=0, \quad \tau_{2} \\
=15, \quad \tau_{3}=0, \quad \tau_{4}=0 .
\end{gathered}
$$

2. By applying algorithm 2 function $R(\tilde{A})=$ $\frac{w}{4}[a+2 b+c], w \in[0,1]$ then the multiple objective as follows: -

$$
\begin{gathered}
\text { Max } z_{1}=0.3125 \tau_{1}+0.1125 \tau_{2}+0.2125 \tau_{3} \\
\quad+0.1125 \tau_{4} \\
\text { Max } z_{2}=0.1125 \tau_{1}-0.1125 \tau_{2}+0.2125 \tau_{3} \\
\quad+0.4125 \tau_{4} \\
\text { Max } z_{3}=-0.1125 \tau_{1}+0.5125 \tau_{2}+0.1125 \tau_{3} \\
\quad+0.2125 \tau_{4}
\end{gathered}
$$

Subject to

$2 \tau_{1}+\tau_{2}+4 \tau_{3}+3 \tau_{4} \leq 60$

$3 \tau_{1}+4 \tau_{2}+\tau_{3}+2 \tau_{4} \leq 60$

$\tau_{1}, \tau_{2}, \tau_{3}, \tau_{4} \geq 0$

Accordingly, when $w \in[0,1]$ we state all the values of $w$ to get the solution which scheduling in Table 1:- 
Table 1. The optimal solution of algorithm 2.

\begin{tabular}{|c|c|c|c|}
\hline \multirow[t]{2}{*}{ Weight } & \multicolumn{3}{|c|}{$\begin{array}{c}\text { Value of the multiple objective } \\
\text { function }\end{array}$} \\
\hline & $z_{1}$ & $z_{2}$ & $z_{3}$ \\
\hline 0.1 & 6.90 & 8.25 & 7.6875 \\
\hline 0.2 & 13.8 & 16.5 & 15.3750 \\
\hline 0.3 & 20.7 & 24.75 & 23.0625 \\
\hline 0.4 & 27.6 & 33. & 30.75 \\
\hline 0.5 & 34.5 & 41.25 & 38.4375 \\
\hline 0.6 & 41.4 & 49.5 & 46.1250 \\
\hline 0.7 & 48.3 & 57.75 & 53.8125 \\
\hline 0.8 & 55.2 & 66 & 61.5 \\
\hline 0.9 & 62.1 & 74.25 & 69.1875 \\
\hline 1 & 69 & 82.5 & 76.8750 \\
\hline
\end{tabular}

The best weight which give us the maximum multiple objective function are $w=1$, that means when $w=1$ then the Eq. 4 equal to Eq.6.

\section{Example 2:}

Min $z_{1}=6 \tau_{1}+2 \tau_{2}+3 \tau_{3}$

Min $z_{2}=5 \tau_{1}+\tau_{2}+2 \tau_{3}$

subject to

$2 \tau_{1}-\tau_{2}+2 \tau_{3} \geq 4$

$\tau_{1} \quad+4 \tau_{3} \geq 4$

$\tau_{1}+3 \tau_{2}+2 \tau_{3} \geq 7$

$\tau_{1}, \tau_{2}, \tau_{3} \geq 0$

First: When we solve the crisp (MOP) problem one can obtain the following optimal solution

$$
\begin{array}{rrr}
\text { Min } z_{1}=8.62, \quad \tau_{1}=0, & \tau_{2}=0.75, \\
\text { Min } z_{2}=5.5, \quad \tau_{3}=2.375 . & \tau_{1}=0, & \tau_{2}=0.75, \\
=2.375 . &
\end{array}
$$

Second: Transforming the crisp multiple objective to fuzzy multiple objective and then solve them by two different ranking functions.

Now, we consider the multiple objectives as triangular fuzzy numbers in the problem as follows:

Let $\Delta_{1}=0.8$ and $\Delta_{2}=1.5$ where $\left(c_{i j}-\Delta_{1}, c_{i j}\right.$, $\left.c_{i j}+\Delta_{2}\right), i, j=1,2,3$.

$$
\begin{gathered}
\text { Min } z_{1}=(5.2,6,7.5) \tau_{1}+(1.2,2,3.5) \tau_{2} \\
+(2.2,3,4.5) \tau_{3} \\
\text { Min } z_{2}=(4.2,5,6.5) \tau_{1}-(0.2,1,2.5) \tau_{2} \\
+(1.2,2,3.5) \tau_{3}
\end{gathered}
$$

subject to

$2 \tau_{1}-\tau_{2}+2 \tau_{3} \geq 4$

$\tau_{1}+4 \tau_{3} \geq 4$

$\tau_{1}+3 \tau_{2}+2 \tau_{3} \geq 7$

$\tau_{1}, \tau_{2}, \tau_{3} \geq 0$

1. By applying algorithm 1 function $R(\tilde{A})=$ $\frac{1}{4}[a+2 b+c]$ then the multiple objective as follows:

$\operatorname{Min} z_{1}=6.175 \tau_{1}+2.175 \tau_{2}+3.175 \tau_{3}$ Min $z_{2}=5.175 \tau_{1}+1.175 \tau_{2}+2.175 \tau_{3}$ subject to

$$
\begin{aligned}
& 2 \tau_{1}-\tau_{2}+2 \tau_{3} \geq 4 \\
& \tau_{1}+4 \tau_{3} \geq 4 \\
& \tau_{1}+3 \tau_{2}+2 \tau_{3} \geq 7 \\
& \tau_{1}, \tau_{2}, \tau_{3} \geq 0
\end{aligned}
$$

Solving the crisp multi objective to get the optimal solution

$$
\begin{gathered}
\text { Min } z_{1}=9.1719, \quad \tau_{1}=0, \quad \tau_{2}=0.75, \\
\tau_{3}=2.375 . \\
\text { Min } z_{2}=6.0469, \quad \tau_{1}=0, \\
\tau_{3}=2.375 .
\end{gathered}
$$

2. By applying algorithm 2 function $R(\tilde{A})=$ $\frac{w}{4}[a+2 b+c], w \in[0,1]$ then the multiple objective as follows: -

Min $z_{1}=0.6175 \tau_{1}+0.2175 \tau_{2}+0.3175 \tau_{3}$ Min $z_{2}=0.5175 \tau_{1}+0.1175 \tau_{2}+0.2175 \tau_{3}$ Subject to

$$
\begin{aligned}
& 2 \tau_{1}-\tau_{2}+2 \tau_{3} \geq 4 \\
& \tau_{1}+4 \tau_{3} \geq 4 \\
& \tau_{1}+3 \tau_{2}+2 \tau_{3} \geq 7 \\
& \tau_{1}, \tau_{2}, \tau_{3} \geq 0
\end{aligned}
$$

Accordingly, when $w \epsilon[0,1]$ we state all the values of $w$ to get the solution which scheduling in Table 2: -

Table 2. The optimal solution of algorithm 2.

\begin{tabular}{ccc} 
Weight & \multicolumn{2}{c}{ Value of the multiple objective function } \\
\cline { 2 - 3 } & $\boldsymbol{z}_{\mathbf{1}}$ & $\boldsymbol{z}_{\mathbf{2}}$ \\
0.1 & 0.9172 & 0.6047 \\
0.2 & 1.8344 & 1.2094 \\
0.3 & 2.7516 & 1.8141 \\
0.4 & 3.6688 & 2.4188 \\
0.5 & 4.5859 & 3.0234 \\
0.6 & 5.5031 & 3.6281 \\
0.7 & 6.4203 & 4.2328 \\
0.8 & 7.3375 & 4.8375 \\
0.9 & 8.2547 & 5.4422 \\
1 & 9.1719 & 6.0469 \\
\hline
\end{tabular}

The best weight which give us the largest multiple objective function are $w=1$, that means when $w=$ 1 then the Eq. 4 equal to Eq. 6.

\section{Conclusions:}

In this paper, a fuzzy variable in (MOP) problems is considered with fuzzy variables of multiple objectives. Then two examples are solved, the first one is maximized and second one is minimized. The first example applies the traditional programming model and ordinary ranking function, the second example applies the traditional programming model and weighted ranking function. Finally, it is noted that the ordinary ranking function is equal to weighted ranking function when 
$w=1$, this means that both ranking functions are the best optimal solution.

\section{Authors' declaration:}

- Conflicts of Interest: None.

- We hereby confirm that all the Figures and Tables in the manuscript are mine ours. Besides, the Figures and images, which are not mine ours, have been given the permission for republication attached with the manuscript.

- Ethical Clearance: The project was approved by the local ethical committee in University of Technology.

\section{References:}

1. Bellman R E, Zadeh L A. Decision Making in Fuzzy Environment. Manage Sci. 1970; 17: B141-B164.

2. Zimmermann H J. Fuzzy programming and Linear Programming with Several Objective Functions. FUZZY SET SYST. 1987; 1: 45-55.

3. Maleki H R. Ranking Functions and their Applications to Fuzzy Linear Programming. FJMS. 2002; 4: 283301.

4. Hussein I H, Dheyab A H. A New algorithm using ranking function to find solution for fuzzy transportation problem. IJMSS. 2015; 3(3): 21-26.

5. Nehi $\mathrm{H} \mathrm{M}$, Hajmohamadi H. A ranking function method for solving fuzzy multi-objective linear programming problem. AFMI. 2012; 3(1): 31-38.
6. Porcheivi RS, Vasanthi L. Solving A Multi Objective Fuzzy Variable Linear programming Problem. using Ranking Functions. IJSR. 2013; 2: 733-735.

7. Kiruthiga M, Loganathan C. Fuzzy Multi-Objective Linear Programming Problem Using Fuzzy Programming Model. IJSETR. 2015; 4: 2681-2686.

8. Mitlif R J. A New Method for Solving Fully Fuzzy Multi-Objective Linear Programming Problems. IJS. 2016; 57(3C): 2307-2311.

9. Hussein I H, Abood Z S. Solving Fuzzy Games Problems by Using Ranking Functions. Baghdad Sci J. 2018; 15(1): 98-101.

10. Ingle S M, Ghadle K P. Solving FFLPP Problem with Hexagonal Fuzzy Numbers by New Ranking Method. IJAER. 2019; 14(1): 97-101.

11. Gass I S. Linear Programming Methods and Applications. 5 th ed. St. Louis San Francisco. New York: Dover Publications. 2010.

12. Pradhan A, Biswal M P. Linear Programming Problems with some Multi- Choice Fuzzy Parameters. YUJOR. 2018; 2: 249-264.

13. Nasseri S H, Mahmoudi F. A New Approach to Solve Fully fuzzy Linear Programming Problem. JARIE. 2019; 6(2): 139-149.

14. Pramy F A. An Approach for Solving Fuzzy MultiObjective Linear Fractional Programming Problems, IJMEMS. 2018; 3(3): 280-293.

15.Dinagar D S , Jeyavuthin M M . Fully Fuzzy Integer Linear Programming Problems Under Robust Ranking Techniques. IJMAA.2018; 6(3): 19-25.

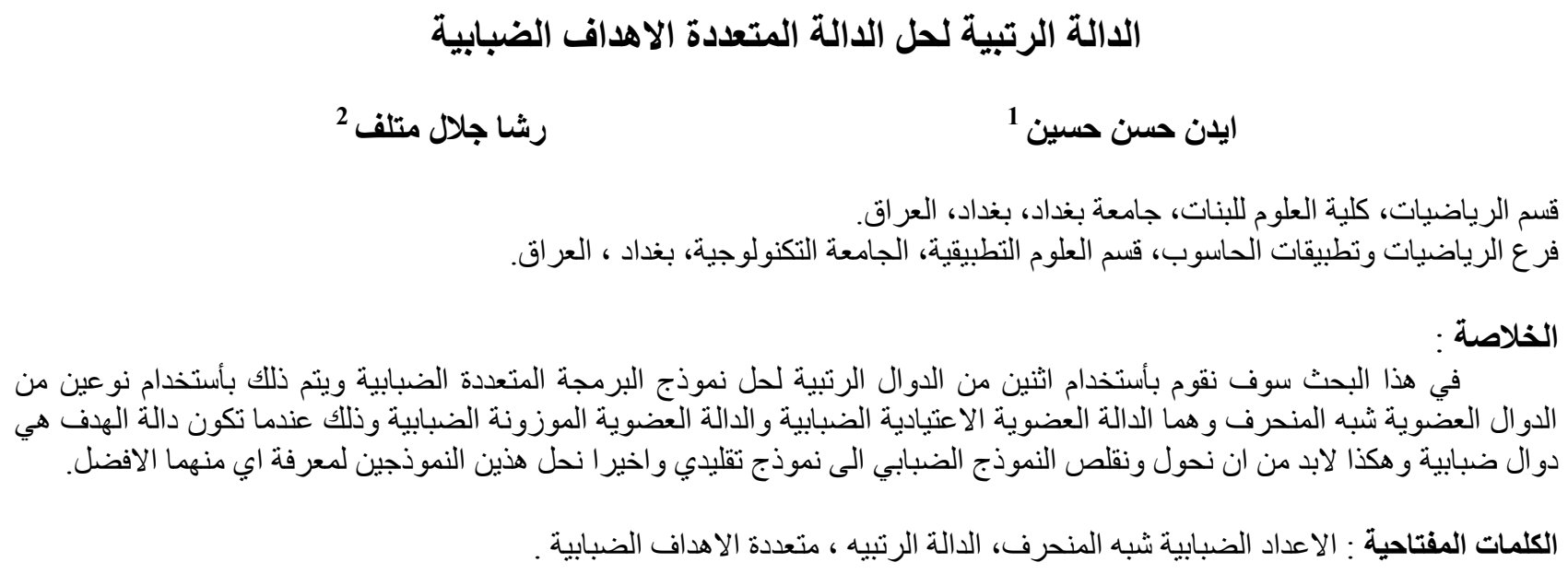

